\title{
PLEURAL SPACE PERFUSION WITH CISPLATIN IN THE MULTIMODALITY TREATMENT OF MALIGNANT MESOTHELIOMA: A FEASIBILITY AND PHARMACOKINETIC STUDY
}

Giovanni B. Ratto, $\mathrm{MD}^{\mathrm{a}}$ Dario Civalleri, MD $^{\mathrm{b}}$

Mauro Esposito, $\mathrm{PhD}^{\mathrm{c}}$

Elisabetta Spessa, MD ${ }^{\mathrm{a}}$

Antonella Alloisio, $\mathrm{MD}^{\mathrm{a}}$

Franco De Cian, MD $^{\mathrm{b}}$

Maria O. Vannozzi, $\mathrm{PhD}^{\mathrm{c}}$
Introduction: Malignant pleural mesothelioma is an ideal model for testing new locoregional multimodality approaches because of its aggressive local behavior. Methods: This study was planned to investigate the feasibility, safety, and pharmacokinetics of a multimodality therapy including an operation, pleural space perfusion (60 minutes) with cisplatin $\left(100 \mathrm{mg} / \mathrm{m}^{2}\right)$, hyperthermia $\left(41.5^{\circ} \mathrm{C}\right)$, and postoperative radiotherapy ( $55 \mathrm{~Gy}$ to chest wall incisions). The effects of the extent of resection and perfusion temperature on cisplatin pharmacokinetics were evaluated. Ten patients with epithelial or mixed, stage I or II, malignant pleural mesothelioma underwent the following procedures: group A (3 patients), pleurectomy/decortication and normothermic pleural space antineoplastic perfusion; group B (3 patients), pleurectomy/decortication and hyperthermic perfusion; and group $C$ (4 patients), pleuropneumonectomy and hyperthermic perfusion. Operations were selectively applied depending on tumor extent. Platinum levels were serially measured by atomic absorption in systemic blood, perfusate, lung, and endothoracic fascia. Results: The overall procedure was completed in every case, without any death or toxicity. No lung damage was demonstrated after treatment. Major complications included 1 wound infection and 1 diaphragmatic prosthesis displacement. The mean peak platinum plasma levels were reached within 45 to 60 minutes after perfusion was started. Systemic drug concentrations were greater after pleurectomy/decortication than after pleuropneumonectomy $(P=.006)$. The local tissue/perfusate ratio of platinum concentrations tended to be higher after hyperthermic perfusion rather than normothermic perfusion. Conclusion: This multimodality approach is feasible, pharmacokinetically advantageous, and safe enough to undergo further clinical investigations. (J Thorac Cardiovasc Surg 1999;117:759-65)
The presently available single-modality or bimodality therapeutic options for malignant pleural mesothelioma have not been proved to yield a significant survival advantage. ${ }^{1}$ Thus there is an urgent need for investigating new multimodality approaches. In planning

From the Istituto Anatomia Chirurgica ${ }^{a}$ and Istituto Clinica Chirurgica, ${ }^{\text {b }}$ University of Genoa; Istituto Nazionale per la Ricerca sul Cancro, ${ }^{\mathrm{c}}$ Servizio di Farmacologia Tossicologica, Genoa, Italy.

Received for publication April 27, 1998; revisions requested July 7, 1998; revisions received Sept 8, 1998; accepted for publication Dec 10, 1998.

Address for reprints: Giovanni B. Ratto, MD, Istituto di Anatomia Chirurgica, Largo Rosanna Benzi 8, Università di Genova, 16132 Genova, Italy.

Copyright $@ 1999$ by Mosby, Inc.

0022-5223/99 $\$ 8.00+0 \quad \mathbf{1 2 / 1 / 9 6 5 3 4}$ combined treatments, an operation remains a suitable therapeutic tool, owing to the limited efficacy of both radiotherapy and systemic chemotherapy. However, it has been stressed that an operation, regardless of whether pleuropneumonectomy or pleurectomy/decortication is performed, basically represents a cytoreductive procedure, requiring adjuvant therapies. ${ }^{2}$ In this setting, malignant mesothelioma appears to be an ideal model for the application of regional chemotherapy because of its tendency to remain, at least initially, a local disease. ${ }^{3}$ The feasibility and safety of intrapleural cisplatin-based chemotherapy has already been demonstrated $^{4}$ with different therapeutic approaches. Normothermic intrapleural antineoplastic instillation has been used either after an operation ${ }^{4,5}$ or as a primary treatment. ${ }^{6}$ Hyperthermic pleural space antineoplastic perfusion has been used in patients who did not undergo an 
operation. ${ }^{7,8}$ A multimodality approach including an operation, antineoplastic pleural space perfusion, and hyperthermia was only applied in 4 patients who underwent pleurectomy for either malignant mesothelioma or adenocarcinoma. ${ }^{9}$ In the present study, such a combined approach was used in patients who had undergone either pleurectomy/decortication or pleuropneumonectomy for malignant pleural mesothelioma. The aim of the study was to assess the feasibility and safety of the overall procedure and the effects of both the extent of resection and the application of hyperthermia on the antineoplastic drug pharmacokinetics.

\section{Methods}

Patients. Criteria for selection were as follows: (1) diagnosis of epithelial or mixed malignant mesothelioma, as demonstrated by histologic findings, immunohistochemistry, and, in selected cases, electron microscopy; (2) aged less than 70 years; (3) Eastern Cooperative Oncology Group performance status less than 1 ; (4) no previous treatment; (5) cardiac (ejection fraction $>45 \%$ ) and pulmonary function (predicted postoperative forced expiratory volume in 1 second $>1 \mathrm{~L}$ ) adequate for the planned operation; (6) clinical stages I or II (T1-2 N0); (7) completely resectable tumor, either by pleurectomy/decortication or pleuropneumonectomy; (8) adequate renal, hematologic, and cardiac function; and (9) informed consent. Complete resectability was predicted according to the criteria previously outlined by Sugarbaker and colleagues. ${ }^{1,3}$ Chest wall was preoperatively judged to be invaded if the fat plane between the parietal pleura and the endothoracic fascia appeared to be obliterated on computed tomographic (CT) scans. The protocol was approved by our Institutional Review Board and by the Ethics Committee of the National Cancer Institute of Genoa, and all the procedures were carried out in accordance with the declaration of Helsinki.

Preoperative staging included a CT scan of the chest and abdomen, a ventilation/perfusion lung scan, a cardiopulmonary function evaluation, thoracoscopy, and bronchoscopy. Thoracoscopic incisions were placed in line with the planned subsequent thoracotomy. Mediastinoscopy was only used when CT scan showed enlarged $(>1 \mathrm{~cm})$ mediastinal nodes. None of the patients included in the present study had positive mediastinoscopy.

During the period 1995 to 1997,10 patients who were referred with clinically suspected or pathologically diagnosed malignant mesothelioma were enrolled in the study. Patients underwent the following procedures: group A $(\mathrm{n}=3)$, pleurectomy/decortication, normothermic pleural space perfusion with cisplatin $\left(100 \mathrm{mg} / \mathrm{m}^{2}\right)$, and postoperative radiotherapy; group $\mathrm{B}(\mathrm{n}=3)$, pleurectomy/decortication, hyperthermic $\left(41.5^{\circ} \mathrm{C}\right)$ pleural space perfusion with cisplatin $(100$ $\left.\mathrm{mg} / \mathrm{m}^{2}\right)$, and postoperative radiotherapy; group $\mathrm{C}(\mathrm{n}=4)$, pleuropneumonectomy, hyperthermic $\left(41.5^{\circ} \mathrm{C}\right)$ pleural space perfusion with cisplatin $\left(100 \mathrm{mg} / \mathrm{m}^{2}\right)$, and postoperative radiotherapy. There were 9 male patients and 1 female patient, ranging in age from 42 to 67 years. The cell type was epithelial in 4 patients and mixed in 6 patients.

Operation. Because the value of pleuropneumonectomy with respect to pleurectomy/decortication remains uncertain, ${ }^{10,11}$ the 2 surgical options were selectively applied on the basis of lung involvement and patient cardiorespiratory function. The aim of the operation was to achieve a macroscopically complete resection, sparing as much apparently normal lung tissue as possible. Patients scheduled to have pleurectomy/decortication were randomly assigned to the normothermic or hyperthermic groups.

Before the operation, a thermodilution catheter (SwanGanz; Baxter Healthcare Corp, Edwards Division, Santa Ana, Calif) was positioned into the pulmonary artery to monitor hemodynamic changes and central temperature. A posterolateral thoracotomy sparing the serratus anterior muscle, with subperiosteal resection of the fifth rib, was routinely used. Whenever lung involvement appeared to be limited, pleurectomy with en bloc minimal wedge resections of the involved parenchyma and removal of the hemidiaphragm and pericardium was applied. Pleuropneumonectomy included en bloc resection of the parietal and visceral pleura, lung, ipsilateral hemidiaphragm, and pericardium. ${ }^{10,12}$ To accurately dissect and resect the entire diaphragm, thoracoscopic optics and instruments were used. In 3 cases in which diaphragm resection proved to be laborious, a counterincision was associated to expose the costophrenic sulcus. Previous thoracoscopic incisions were removed by means of limited chest wall resections. When the diaphragm was resected, care was taken to preserve the underlying peritoneum. In the left side, the diaphragm was always replaced with an expanded polytetrafluoroethylene membrane; in the right side, the prosthesis was applied only when the peritoneum had been withdrawn with the diaphragm. The pericardium was reconstructed with a expanded polytetrafluoroethylene prosthetic patch in all cases.

Pleural space perfusion. A standard cisplatin dose (100 $\mathrm{mg} / \mathrm{m}^{2}$ ) with systemic prehydration was used for the known safety of intrapleural ${ }^{6}$ and intrapulmonary ${ }^{13}$ cisplatin and its reported activity in both pleural ${ }^{14}$ and peritoneal ${ }^{15}$ malignant mesothelioma. No dose escalation was scheduled because very high local concentrations of platinum were expected even with standard dosages, whereas lower doses should have exposed patients to suboptimal drug concentrations. Pleural space perfusion was begun after the completion of the operation and meticulous chest wall closure. Inflow chest drains (2 tubes in pleurectomy/decortication and 1 tube in pleuropneumonectomy) were positioned at the bottom of the pleural cavity. An additional outflow tube was inserted at the top of the pleural cavity. The tubes were connected to a perfusion circuit consisting of polyvinylchloride tubing, a heat exchanger, and a roller pump. Because a temperature of $44^{\circ} \mathrm{C}$ to $45^{\circ} \mathrm{C}$ on the heat exchanger was selected, the minimal and maximal pleural space perfusion temperatures were maintained between $41.5^{\circ} \mathrm{C}$ and $42^{\circ} \mathrm{C}$. They were monitored with thermoprobes positioned in the pleural cavity. The body temperature was continuously checked with an esophageal and a rectal probe. 


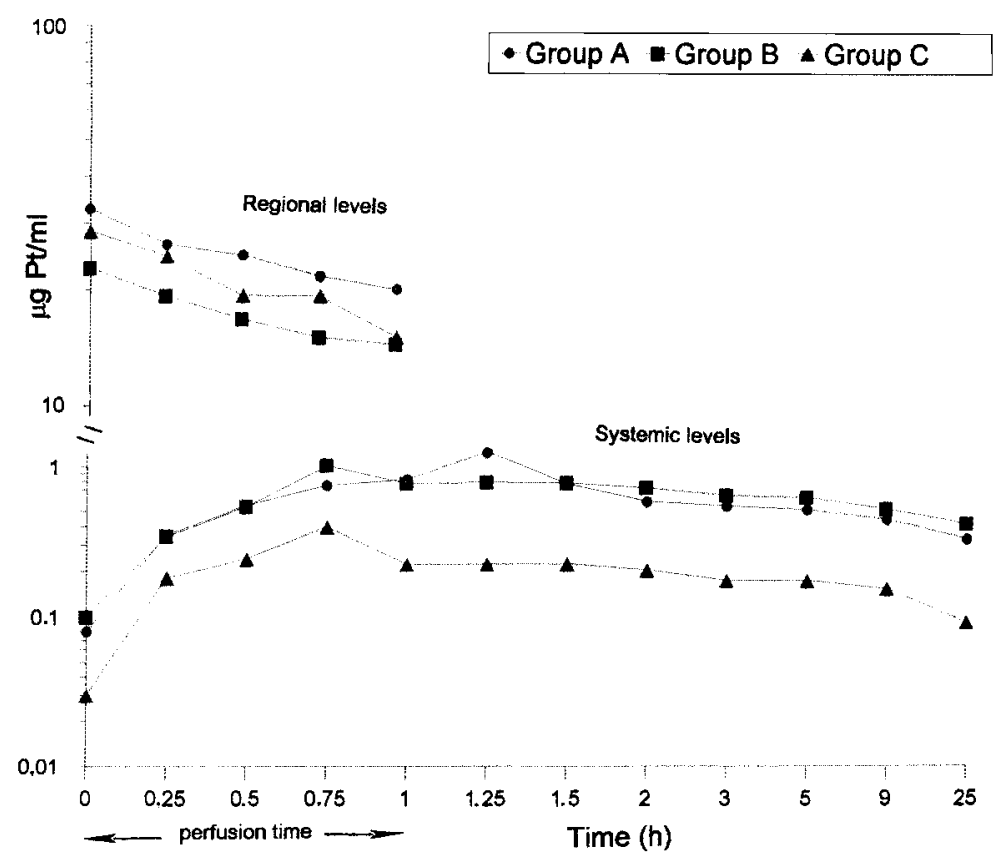

Fig 1. Semilogarithmic plot of systemic and regional (pleural space perfusate). Platinum concentrations versus time in patients with malignant mesothelioma who underwent pleurectomy/decortication and normothermic pleural space perfusion with cisplatin (group A; O); pleurectomy/decortication and hyperthermic pleural space perfusion with cisplatin (group B; $\mathbf{\square}$ ); pleuropneumonectomy and hyperthermic pleural space perfusion with cisplatin (group C; $\mathbf{\Delta}$ ).

No prednisolone for prevention of pulmonary edema was given. ${ }^{8}$ The circuits were primed with a mean volume of $4 \mathrm{~L}$, as previously described. ${ }^{13}$ The pleural space perfusion flow was maintained between 200 and $300 \mathrm{~mL} / \mathrm{min}$, as monitored with an electromagnetic flow probe. Chest cavity perfusion was carried out for 60 minutes. At the end of the perfusion, the perfusate was discarded. All patients received preventive antiemetics and were evaluated for toxicity at 12-hour intervals. Approximately 30 days after the treatment, patients received 55 Gy of radiotherapy to the thoracotomic incision and sites of chest tubes. As previously described, ${ }^{13}$ simultaneous samples for analysis of platinum concentrations were drawn from the perfusion circuit and general circulation before drug administration and at $0,5,15,30,45$, and $60 \mathrm{~min}-$ utes after cisplatin perfusion was started. Systemic blood samples were also collected at 15 and 30 minutes and at 1, 2, 4, 8, and 24 hours after the treatment. At the end of the perfusion, lung parenchyma and endothoracic fascia samples were obtained for morphologic examination and platinum level measurements (only fascia samples in the pleuropneumonectomy group). Platinum analysis in fluids and tissues and morphologic evaluations were performed according to methods previously described in detail. ${ }^{13}$ The area under the concentration multiplied by the time curve (AUC) from 0 to 60 minutes was estimated by the trapezoidal rule. Follow-up evaluation, including high resolution CT scan, lung volume, and airflow measurements, and determination of pulmonary diffusing capacity and arterial blood gas values were scheduled at 3, 6, and 12 months after treatment and yearly thereafter.

\section{Results}

The operation was completed in every case without serious complications, and no macroscopic residual disease was left within the chest at the end of the procedure. During perfusion, the maximum temperature in the thoracic esophagus did not exceed $38^{\circ} \mathrm{C}$, and no changes in hemodynamic or metabolic parameters and in blood gas values were found. There was no postoperative death. Major postoperative complications included 1 wound infection and 1 diaphragmatic prosthesis displacement. The last patient required an emergency second operation. Drug toxicity, such as nausea, vomiting, myelosuppression, or neuropathy, did not occur. Transient postoperative elevation of creatinine values (maximal level, $1.4 \mathrm{mg} / \mathrm{dL}$ ) was found in 4 patients. Total platinum concentrations in the circuit fluid and in systemic plasma are reported in Fig 1. The data reported in Table I on total systemic and regional exposure to platinum during perfusion (AUC, 0-60 minutes) and their mean ratio (AUC regional/AUC systemic values) 
Table I. Systemic and regional exposure (AUC) to total platinum during pleural space perfusion

\begin{tabular}{llll}
\hline & Group $A^{*}$ & Group B† & Group C $\ddagger$ \\
\hline $\begin{array}{c}\text { AUC plasma } \\
(\mu \mathrm{g} \times \mathrm{min} / \mathrm{mL})\end{array}$ & $31.2 \pm 5.9$ & $34.6 \pm 17.0$ & $14.1 \pm 2.9$ \\
$\begin{array}{c}\text { AUC regional } \\
(\mu \mathrm{g} \times \mathrm{min} / \mathrm{mL})\end{array}$ & $1476 \pm 181$ & $1036 \pm 200$ & $1218 \pm 187$ \\
$\begin{array}{c}\text { AUC regional/ } \\
\text { AUC plasma ratio }\end{array}$ & $49.1 \pm 4.6$ & $31.8 \pm 8.6$ & $87.9 \pm 6.2 \S$ \\
\hline
\end{tabular}

Values are given as mean \pm SE.

*Pleurectomy/decortication (normothermic perfusion)

$\dagger$ Pleurectomy/decortication (hyperthermic perfusion).

$\ddagger$ Pleuropneumonectomy (hyperthermic perfusion).

$\S P=.006$ (Student $t$ test) versus group B.

show that systemic absorption of cisplatin from the circuit was greater in patients undergoing pleurectomy/ decortication (group B) than in those patients who underwent pleuropneumonectomy (group C); the mean \pm SE AUC regional/AUC systemic values were $31.8 \pm$ 8.6 and $87.9 \pm 6.2$, respectively $(P=.006)$. Moreover, hyperthermia tended to enhance cisplatin uptake from the circuit, as suggested by the mean \pm SE AUC regional/AUC systemic ratio. The relevant values in patients with pleurectomy/decortication were $49.1 \pm 4.6$ in normothermic conditions (group A) versus $31.8 \pm 8.6$ in hyperthermic conditions (group B). In the 3 groups of patients, mean peak platinum plasma levels were reached within 45 to 60 minutes of drug administration and tended to be higher in group B $(1.00-0.43 \mu \mathrm{g} / \mathrm{mL})$ than in group $\mathrm{C}(0.39-0.10 \mu \mathrm{g} / \mathrm{mL})$, whereas mean circuit platinum concentrations were similar (Fig 1). Total platinum concentrations in lung parenchyma and endothoracic fascia are reported in Table II. In a previous experimental study from our laboratory, pigs received a similar dose of cisplatin intravenously ${ }^{16}$; platinum concentrations in lung parenchyma of the patients undergoing pleural space perfusion were nearly 5 times as high as platinum concentrations in the lung of pigs. At the end of the 1-hour perfusion, the patient tissue/patient perfusate ratio tended to be higher in patients treated with hyperthermic perfusion than in those patients treated with normothermic perfusion (group B vs group A). The mean patient lung/patient perfusate ratio values were 0.35 for group $B$ and 0.25 for group A, respectively. The mean patient endothoracic fascia/patient perfusate ratio values were 0.31 and 0.19 for group B and A, respectively. Conversely, the patient endothoracic fascia/patient perfusate ratio values were similar in the 2 patient groups who underwent hyperthermic perfusion, 0.31 for group B and 0.37 for group C.
Table II. Mean total platinum concentrations in tissues at the end of 1 hour of perfusion

\begin{tabular}{lccc}
\hline & Group $A^{*}$ & Group B $\dagger$ & Group $C \ddagger$ \\
\hline Lung $(\mu \mathrm{g} \mathrm{Pt} / \mathrm{g})$ & $5.15 \pm 1.58$ & $5.25 \pm 1.50$ & \\
Endothoracic fascia & $3.96 \pm 1.34$ & $4.68 \pm 1.42$ & $5.24 \pm 0.64$ \\
$\quad(\mu \mathrm{g} \mathrm{Pt} / \mathrm{g})$ & & & \\
Lung/regional ratio & $0.25 \pm 0.05$ & $0.35 \pm 0.05$ & \\
Endothoracic fascia/ & $0.19 \pm 0.04$ & $0.31 \pm 0.05$ & $0.37 \pm 0.01$ \\
$\quad$ regional ratio & & & \\
\hline
\end{tabular}

Values are given as mean $\pm \mathrm{SE} ; \mu g \mathrm{Pt} / \mathrm{g}$, micrograms platinum per gram of tissue.

*Pleurectomy/decortication (normothermic perfusion).

$\dagger$ Pleurectomy/decortication (hyperthermic perfusion).

$\$$ Pleuropneumonectomy (hyperthermic perfusion).

Morphologic examination of lung parenchyma showed no damage as a result of pleural cavity perfusion, either normothermic or hyperthermic (Fig 2). Electron microscopic examination showed no abnormality of both the alveolar septa and epithelial lining. Follow-up evaluation did not show significant changes in the interstitium of treated lungs (Fig 3, $A$ and $B$ ). The post-treatment sequential assessment of respiratory function parameters demonstrated consistent worsening in only 3 patients at disease progression.

\section{Discussion}

Sugarbaker and colleagues ${ }^{1}$ have recently emphasized the concept that a multimodality approach has the potential to enhance clinical results of malignant pleural mesothelioma therapy. Because malignant mesothelioma has an aggressive local behavior, ${ }^{1,17}$ multimodality regimens should include locoregional treatments aimed to improve local control. ${ }^{10}$ Intrapleural drug instillation has been used either as primary procedure ${ }^{6}$ or after an operation. ${ }^{4,5,18,19}$ In both instances, cisplatin instillation proved to be feasible and well tolerated. The pharmacokinetics of both cisplatin and mitomycin have been explored in 12 patients with malignant mesothelioma who underwent operation and immediate intrapleural antineoplastic instillation. ${ }^{4}$ Although peak plasma levels approximated those attained after systemic infusion, the peaks were delayed, and a 3- to 5-fold advantage for pleural to plasma AUCs was found. In 2 further studies, intrapleural cisplatin-based normothermic chemotherapy $\left(100 \mathrm{mg} / \mathrm{m}^{2}\right)$ was coupled with an operation. ${ }^{18,19}$ In both series the treatment proved feasible and safe, although median survival remained 11.5 months.

Postoperative antineoplastic pleural space perfusion gives several theoretic advantages over intrapleural drug instillation. The latter, if applied without opera- 


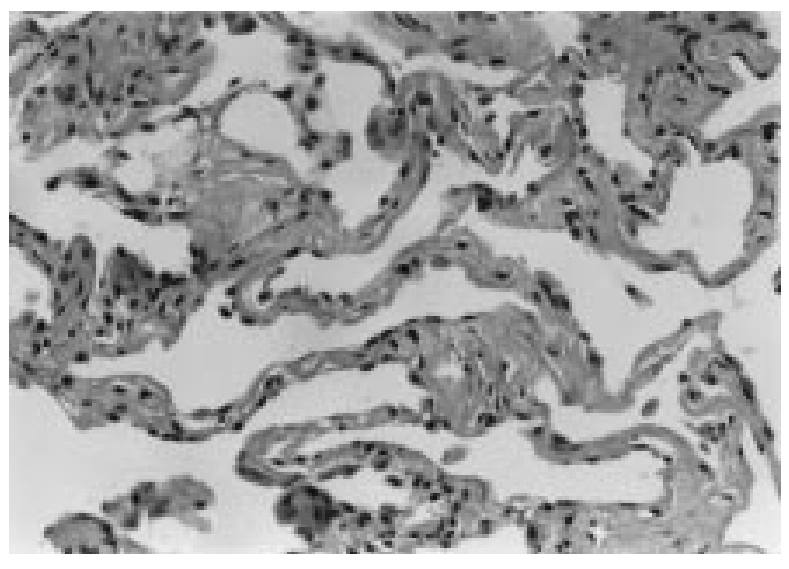

Fig 2. Microscopic appearance of normal lung parenchyma after pleurectomy/decortication followed by 1 hour of hyperthermic pleural space perfusion with cisplatin. (Hematoxylineosin stain; original magnification, $\times 250$.)

tion, requires a free pleural space and minimal tumor burden, because drugs penetrate tumor to a distance of only a few millimeters. ${ }^{14}$ Intracavitary drug instillation, if given immediately after the operation, requires changing the patient's position every 30 minutes to achieve good drug distribution throughout the pleural space. By contrast, perfusion techniques are likely to minimize the risk of local changes in drug absorption and, notably, to provide the best means of coupling chemotherapy and hyperthermia. The application of hyperthermia in a combined antitumor program seems to be attractive because of its effectiveness against malignant cells and its potential to enhance cisplatin cytotoxicity. ${ }^{9,20}$ Both preclinical ${ }^{20,21,22}$ and clinical $^{23}$ studies showed that hyperthermia significantly increases tumor growth delay by enhancing antineoplastic drug uptake and cytotoxicity. Intrapleural perfusion hyperthermic chemotherapy has been used without pleurectomy in 2 studies $^{7,8}$ and after pleurectomy in 1 study. ${ }^{9}$ Perfusions were carried out for time periods ranging from 60 to 120 minutes; temperature was maintained between $42^{\circ} \mathrm{C}$ and $43^{\circ} \mathrm{C}$. No major complications developed, and pharmacokinetic studies showed that a high concentration of cisplatin was retained in the pleural cavity during perfusion.

The present study showed that intrapleural perfusion hyperthermic chemotherapy is technically simple, safe, and pharmacologically advantageous particularly after pleuropneumonectomy. Sugarbaker and colleagues ${ }^{12}$ have already shown that an aggressive multimodality regimen including pleuropneumonectomy may be completed with acceptable morbidity and mortality
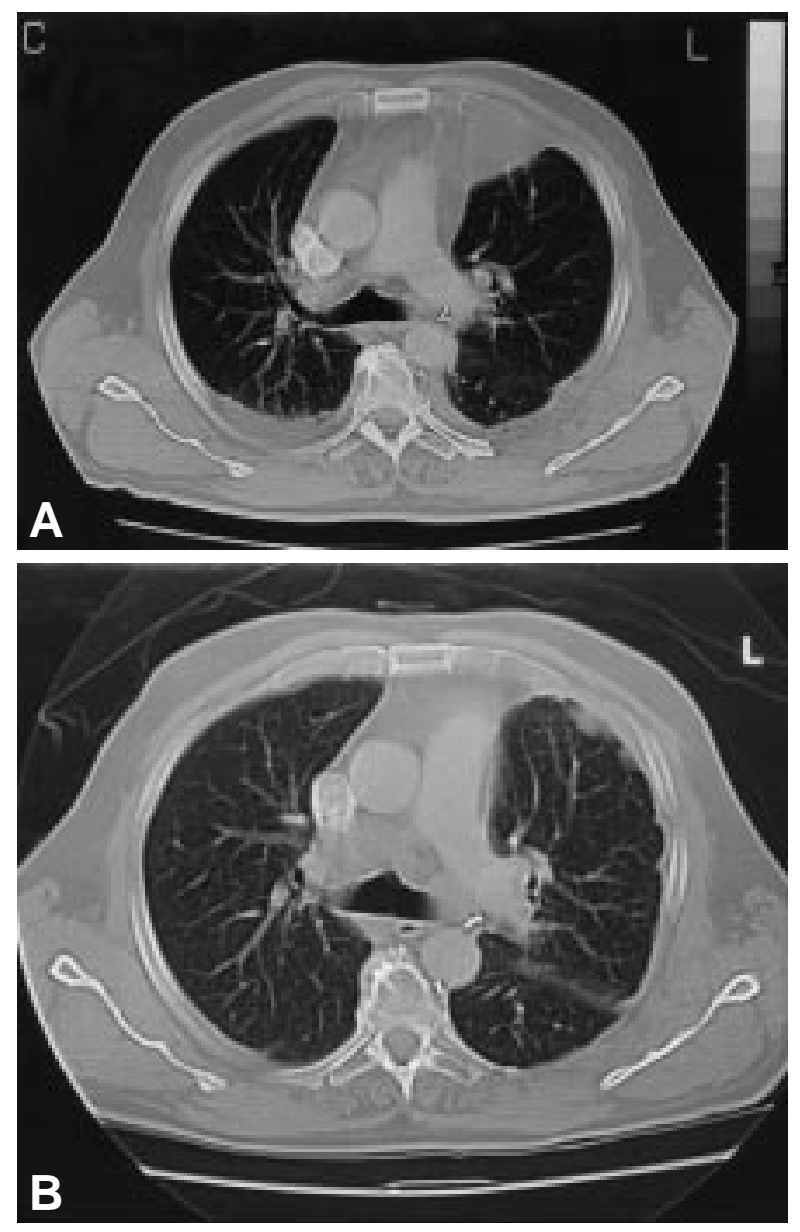

Fig 3. CT scan appearance of the lung parenchyma, 3 months (A) and 12 months (B) after pleurectomy/decortication and hyperthermic pleural space perfusion with cisplatin.

rates $(4.6 \%)$; our experience indicated that the association of pleuropneumonectomy and hyperthermic pleural space perfusion is feasible and safe. Our pharmacokinetic results may have some implications for the clinical application of intracavitary chemotherapy. The first notable finding is the significant plasma concentration of cisplatin after intrapleural administration. This implies that, because of the great drug absorption from the pleural space, a highly toxic agent cannot be proposed for intracavitary administration. Our data are in agreement with those reported by Rusch and colleagues, ${ }^{4}$ indicating that, after decortication, cisplatin is rapidly absorbed from the pleural space and reaches peak plasma concentrations similar to those attained after intravenous infusion, though substantially delayed. Perhaps the outstanding finding from our experience is the more favorable regional to systemic 
plasma platinum concentrations in the pleuropnemonectomy group than in the pleurectomy/decortication group. These data support the hypothesis that the lung plays an important role in cisplatin absorption from the pleural space. Because the therapeutic purpose is to deliver maximum drug concentrations to minimum residual tumor, the present study seems to suggest that pleuropneumonectomy followed by hyperthermic antineoplastic pleural space perfusion is the best multimodality approach. In patients with minimal visceral pleura involvement who are functionally compromised, where pleurectomy/decortication remains the first choice operation, lung exclusion by clamping both pulmonary artery and veins during perfusion could be useful in reducing drug absorption and systemic toxicity. ${ }^{16}$ Our pharmacokinetic data also seem to support an enhanced cisplatin uptake from the pleural space by hyperthermia, because it could be suggested by the higher ratio between platinum concentrations in tissues and in the perfusate. Similarly, Formenti and colleagues $^{24}$ reported an increased platinum clearance in patients with ovarian cancer who received intraperitoneal carboplatin and hyperthermia.

It could be claimed that a pharmacokinetic advantage for cisplatin pleural exposure does not necessarily translate into an enhanced delivery of the drug to target tissues. The present study showed that a high concentration of platinum reached both the lung and the chest wall fascia, which are the organs potentially involved with residual tumor. Whether a higher delivery of the drug to the target organs results in prolonged diseasefree and overall survival remains to be determined. The evaluation of this point is out of the scope of the present study. Even literature analysis does not clarify this topic, because of the lack of randomized trials of adequate size.

Our morphologic and functional investigations showed no lung damage from pleural space perfusion. This is in agreement with the results of Lerza and colleagues ${ }^{6}$ and indicates that the limiting factor in escalating cisplatin doses is systemic toxicity.

In the present series, single-drug chemotherapy has been used, even though no response greater than $20 \%$ could be expected. ${ }^{25}$ Unconfirmed data would suggest that combination chemotherapy including doxorubicin, cisplatin, bleomycin, and mitomycin might achieve a response rate as high as $44 \% .{ }^{25}$ Our perfusion technique may provide the basis for applying potentially more effective combination chemotherapy.

In conclusion, our results indicate that the combinedmodality regimen that includes an operation and pleural space perfusion with cisplatin and hyperthermia is feasible, safe, and pharmacokinetically advantageous. Further advantages of the procedure are its easy technical application, versatility in associating physical therapies, and suitable temperature control. Because of the low prevalence of malignant pleural mesothelioma, multi-institutional prospective trials are required to validate the efficacy of the procedure and to compare distinct pharmacologic regimens.

\section{REFERENCES}

1. Sugarbaker DJ, Garcia JP. Multimodality therapy for malignant pleural mesothelioma. Chest 1997;112:272-5.

2. Rusch VW. Pleurectomy/decortication and adjuvant therapy for malignant mesothelioma. Chest 1993;103:282S-4S.

3. Sugarbaker DJ, Strauss GM, Lynch TO, Richards W, Mentzer SJ, Lee TH, et al. Node status has prognostic significance in the multimodality therapy of diffuse malignant mesothelioma. J Clin Oncol 1993;11:1172-8.

4. Rusch VW, Niedzwiecki D, Tao Y, Menendez-Botet C, Dnistrian A, Kelsen D, et al. Intrapleural cisplatin and mitomycin for malignant mesothelioma following pneumonectomy: pharmacokinetic studies. J Clin Oncol 1992;10:1001-6.

5. Yasumoto K, Shimokawa T, Nagashima A, Hirose N, Nakahashi H. Pharmacokinetics of cisplatin instilled into the pleural cavity following panpleuropneumonectomy in patients with malignant pleurisy due to lung cancer. J Surg Oncol 1993;54:67-70.

6. Lerza R, Esposito M, Vannozzi M, Bottino GB, Bogliolo G, Pannacciulli I. High dose of intrapleural cisplatin in a case of malignant pleural mesothelioma. Cancer 1994;73:79-84.

7. Fujimura T, Yonemura Y, Nojima N, Nakai M, Kaji M, Sahara H, et al. Intrathoracic hyperthermochemotherapeutic perfusion for the intrathoracic malignancies in gastric cancer. Hepatogastroenterology 1995;42:878-84.

8. Matsuzaki Y, Shibata K, Yoshioka M, Inoue M, Sekiya R, Onitsuka T, et al. Intrapleural perfusion hyperthermic chemotherapy for malignant pleural dissemination and effusion. Ann Thorac Surg 1995;59:127-31.

9. Carry PY, Gilly FN, Petit PY, Sayag AC, Petit PY Rochette C, et al. A new device for treatment of pleural malignancies: intrapleural chemohyperthermia preliminary report. Oncology 1993;50:348-52.

10. Rusch VW. Clinical features and current treatment of diffuse malignant pleural mesothelioma. Lung Cancer 1995;12(suppl): 127-46.

11. Pass HI, Krandak K, Temeck BK, Feuerstein I, Steinberg SM. Surgically debulked malignant pleural mesothelioma: results and prognostic factors. Ann Surg Oncol 1997;4:215-22.

12. Sugarbaker DJ, Mentzer SJ, DeCamp M, Lynch TO, Strauss GM. Extrapleural pneumonectomy in the setting of a multimodality approach to malignant mesothelioma. Chest 1993;103:377-81.

13. Ratto GB, Toma S, Civalleri D, Passerone GC, Esposito M, Zaccheo $\mathrm{D}$, et al. Isolated lung perfusion with platinum in the treatment of pulmonary metastases from soft tissue sarcomas. J Thorac Cardiovasc Surg 1996;112:614-22.

14. Rusch VW, Figlin R, Godwin D, Piantadosi S. Intrapleural cisplatin and cytarabine in the management of malignant pleural effusions: a lung cancer study group trial. J Clin Oncol 1991; 9:313-9.

15. Ma GY, Bartlett DL, Reed E, Figg WD, Lush RM, Lee KB. 
Continuous hyperthermic peritoneal perfusion for the treatment of peritoneal mesothelioma. Cancer J Sci Am 1997;3:174-9.

16. Ratto GB, Esposito M, Vannozzi M, Fulco RA, Rovida S. Experimental models of regional chemotherapy via the pulmonary artery using cisplatin. Reg Cancer Treat 1990;3:222-7.

17. Baldini EH, Recht A, Strauss GM, DeCamp MM Jr, Swanson SJ, Liptay MJ, et al. Patterns of failure ofter trimodality therapy for malignant pleural mesothelioma. Ann Thorac Surg 1997;63:334-8.

18. Colleoni M, Sartori F, Calabro F, Nelli P, Vicario G, Sgarbossa G, et al. Surgery followed by intracavitary plus systemic chemotherapy in malignant pleural mesothelioma. Tumori 1996;82:53-6.

19. Lee JD, Perez S, Wang HJ, Figlin RA, Holmes EC. Intrapleural chemotherapy for patients with incompletely resected malignant mesothelioma: the UCLA experience. J Surg Oncol 1995;60:2627.

20. Los G, vanVugt MJH, Pinedo HM. Response to peritoneal solid tumors after intraperitoneal chemohyperthermia treatment with cisplatin and carboplatin. Br J Cancer 1994;69:235-41.

21. Takahashi I, Maehara Y, Kusumoto H, Kusumoto T, Baba H,
Kohnoe S, et al. Interaction of cis-diammine-dichloroplatinum (II) and its analogues cis-1,1-cyclobutanedycarboxylato 2R-2methyl -1,4- butanediammineplatinum (II) and cis-diammine (glycolato) platinum with hyperthermia in vivo. Oncology 1996; 53:68-72.

22. Ohtsubo T, Saito H, Tanaka N, Tsuzuki H, Daito T, Gano E. In vitro effect of hyperthermia on chemoenhancement and uptake of cisplatin in human pharingeal carcinoma KB cells. Chemotherapy 1997;43:43-50.

23. Storm FK, Kaiser LR, Silberman A, Harrison WH, Elliot RS, Morton DL. Thermo-chemotherapy for melanoma metastases in liver. Cancer 1982;49:1243-8.

24. Formenti SC, Shrivastava PN, Sapozink M, Jozsef G, Chan KK, Jeffers $\mathrm{S}$, et al. Abdomino-pelvic hyperthermia and intraperitoneal carboplatin in epithelial ovarian cancer: feasibility, tolerance and pharmacology. Int J Radiat Oncol Biol Phys 1996;35: 993-1001.

25. Ong ST, Vogelzang NJ. Chemotherapy in malignant pleural mesothelioma: a review. J Clin Oncol 1996;14:1007-17.

\section{Bound volumes available to subscribers}

Bound volumes of The Journal of Thoracic and Cardiovascular Surgery are available to subscribers (only) for the 1999 issues from the Publisher, at a cost of $\$ 134.00$ for domestic, $\$ 165.85$ for Canadian, and $\$ 155.00$ for international subscribers for Vol 117 (January-June) and Vol 118 (July-December). Shipping charges are included. Each bound volume contains a subject and author index and all advertising is removed. Copies are shipped within 60 days after publication of the last issue of the volume. The binding is durable buckram with the Journal name, volume number, and year stamped in gold on the spine. Payment must accompany all orders. Contact Mosby, Inc, Subscription Services, 11830 Westline Industrial Drive, St Louis, MO 63146-3318, USA; phone 800-453-4351 or 314-453-4351.

Subscriptions must be in force to qualify. Bound volumes are not available in place of a regular Journal subscription. 